\title{
Effect of Cognitive Load on Tactor Location Identification in Zero-g
}

\author{
Anu Bhargava, Michael Scott, Ryan Traylor, Roy Chung, Kimberly Mrozek, Jonathan Wolter, \\ and Hong Z. Tan \\ Haptic Interface Research Laboratory, Purdue University, West Lafayette, IN, USA \\ \{abhargav,hicsurfr,traylorr,rbchung,mrozekk,jwolter,hongtan\}@purdue.edu
}

\begin{abstract}
A wearable haptic interface has been developed to impart vibrotactile information to its user with the goal of improving situation awareness. The effectiveness of the haptic interface has been evaluated in three experiments aboard the NASA KC-135A reduced gravity aircraft. During the third flight, subjects identified the locations of tactors embedded in the haptic display in a microgravity environment. We report our results on how cognitive load affects one's ability to identify the locations of vibrotactile stimulations in the altered-gravity environment. Cognitive load was manipulated by requiring subjects to be strapped to the floor of the KC-135 (low cognitive-load condition) or allowing them to float freely in microgravity (high cognitive-load condition). It was found that tactor-location identification was more accurate in the low cognitive-load condition than in the high cognitive-load condition. Our results have implications for the design of multimodal user interfaces in general.
\end{abstract}

\section{Introduction}

Our work is motivated by the desire to alleviate spatial disorientation that frequently occurs in alteredgravity environments. We briefly outline the problems associated with spatial disorientation, our previous work on a haptic display for situation awareness, and the goals of current study.

\subsection{Spatial Disorientation}

Spatial disorientation (SD) is a state characterized by an erroneous orientational perception of attitude, altitude, or motion of one's own aircraft relative to the earth or other significant objects [1]. It is a tri-service aviation problem that annually costs the US Department of Defense in excess of $\$ 300$ million in lost aircrafts. Spatial disorientation is the number one cause of pilot-related mishaps in the Navy and the Air Force. A typical SD mishap occurs when the pilot's visual system is compromised by temporary distractions, increased workload, reduced visibility, and most commonly, g-lock, which occurs when the pilot undergoes a high-g maneuver and temporarily blacks out behind the stick [2]. Frequently, after pilots recover from the distraction, they rely on instinct rather than the instrument panel to fly the aircraft. Often, the orientation of the aircraft as perceived by the pilot is much different than the actual orientation of the aircraft. As a result, disaster strikes.

Two common approaches exist towards solving the problem of spatial disorientation. The first is the use of visual orientation cues. For example, at MIT's Man-Vehicle Laboratory, researchers study the physiological and cognitive limitations of pilots and passengers of aircraft and spacecraft to optimize the system's effectiveness and safety. They use techniques from cognitive psychology, sensory-motor physiology and human factors [3]. The second approach is the use of haptic orientation cues. The Tactile Situation Awareness System (TSAS) system, developed at the Naval Aeromedical Research Laboratory, takes data from the aircraft's avionics and relays this information to the pilot via an array of pneumatic tactors integrated into a flight vest [4]. The Cutaneous Communication Laboratory at Princeton University has been researching how the skin may be used to sense patterns based on sensory saltation [5]. Researchers at TNO Human Factors in the Netherlands have successfully demonstrated a way-finding display built into a belt worn around the waist [6].

\subsection{Our Previous Work}

At the Haptic Interface Research Lab at Purdue, we have developed a haptic back display for situation awareness and attention cueing [7]. Variations of this back display have been used onboard NASA's KC- 
135A reduced-gravity aircraft to test its effectiveness in a microgravity environment. The first experiment was conducted in the summer of 1999. It examined how the sense of touch can be engaged in a natural and intuitive manner to allow for correct perception of position, motion and acceleration of one's body in altered gravity environments. Specifically, a 3-by-3 tactor array was used to deliver saltatory signals in the four cardinal directions of left, right, up, and down (see [8] for a review of the sensory saltation phenomenon; see [9] for a brief summary of our first flight). The results showed that whereas subjects could easily identify the four directions with 100\% accuracy in the lab in one-g, the accuracy dropped to $44 \%$ in zero gravity [9]. Anecdotal reports indicated that the vibrotactile stimulation felt much weaker in zero-g than in one-g. It was hypothesized that there might be three possible causes for the decreased performance in zero-g: the dynamics of the tactors might have changed, the perceptual threshold for vibrotactile stimulation might have increased, and cognitive load might have increased due to flying in microgravity.

The second flight in March of 2001 addressed the first two possible causes. In order to determine if the dynamics of the tactors changed during periods of microgravity, an accelerometer was placed on a single tactor attached to the user's wrist and recorded the vibrations on the surface of the tactor in altered-gravity environments. To determine if perceptual threshold increased in microgravity, a psychophysical experiment was conducted to compare perceived intensities of vibrotactile signals occurring during the zero-g and the 1.8-g phases of parabola flights. The results indicated that (1) the tactors were producing the same amount of displacement given the same driving waveform in one-g and zero-g conditions, and (2) the perceived loudness of vibrotactile signals were equivalent in zero-g and 1.8-g environments [9].

\subsection{Aims of Current Study}

This paper reports findings from the third flight that took place in the summer of 2004. This study was designed to test the third and remaining hypothesis that cognitive load affected the perception of vibrotactile stimuli in altered-gravity environment. Students who had flown aboard the KC-135A described how difficult it was to learn to fly in zero-g during the initial parabolas. They discovered new ways of controlling the position and orientation of their bodies in microgravity. It was agreed that one had to pay attention in order not to bump into something or get tangled by a free-flying microphone cord. It was therefore not surprising that the subjects could not devote all their attention to the vibrotactile signals while floating in space. It is well known that dividing attention between two tasks can lead to an increase in cognitive load and a decrease in performance [10].

Two cognitive-load conditions were employed in this study. During the low cognitive-load condition, the subject was strapped to the floor of the KC-135A aircraft so that s/he did not have to worry about body orientation in zero-g. During the high cognitive-load condition, the subject was free-floating in microgravity and had to divide his or her attention between controlling body orientation and attending to tactors. This division of attention was hypothesized to be the main cause of the decreased user performance measured in the first flight.

\section{Methods}

\subsection{Simulation of Altered-gravity}

Altered gravity was simulated by a series of parabolas aboard the KC-135A. Each parabola began with the KC-135A climbing rapidly at a 45 degree angle at approximately 24,000 feet. During the ascent of the plane, a 1.8-g environment was created within the aircraft. This 1.8-g environment had a duration of roughly 25 seconds. Once the plane reached an altitude of 32,000 feet, it began to freefall, thereby creating a microgravity environment inside the aircraft. This microgravity environment had a duration of roughly 25 seconds. After the freefall, the plane began to descend at a 45 degree angle again, completing the parabola. A total of thirty parabolas were flown for this experiment. Data was collected during twenty of the thirty parabolas due to the need to briefly acclimate to the microgravity environment, and the need to prepare for and switch between low and high cognitive-load conditions in the middle of the flight. See http://zerog.jsc.nasa.gov/home.html for further details on how the KC-135A simulates microgravity.

\subsection{Apparatus}

The hardware consisted of four major components: the control keypad, the stimulus generator, the tactor driver circuitry, and the tactile display (Fig. 1). The user interacted with the system via the control keypad. Each key press was sent to an encoding circuitry provided by a 74C922J integrated circuit. The digital output from this chip could be read and interpreted by a microcontroller used for stimulus generation. 


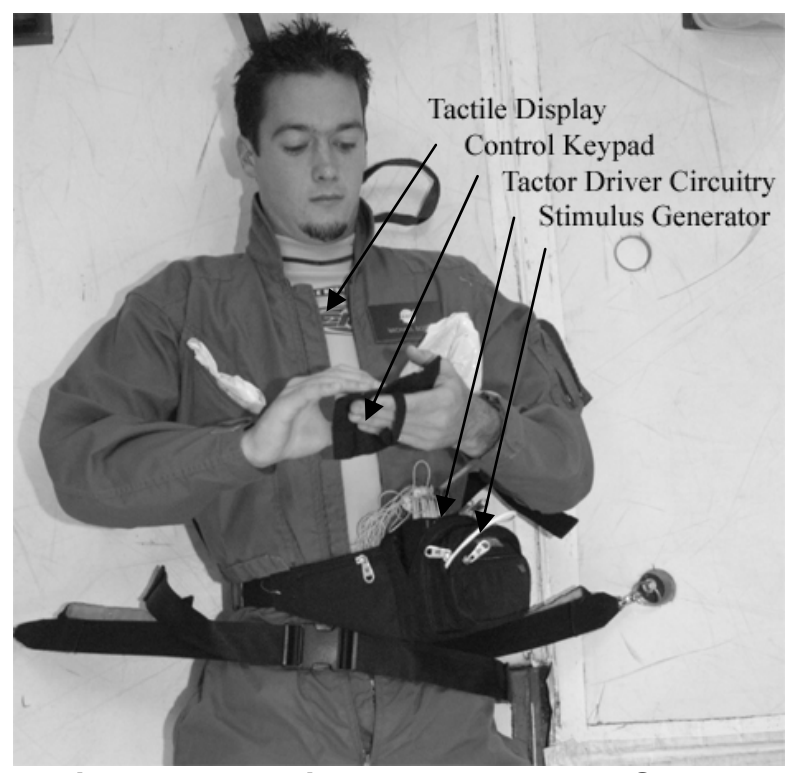

Figure 1. Experimental apparatus. Shown here is one subject conducting the experiment while being strapped to the floor of the KC135A.

The stimulus generator used the AVR ATMEGA16 microcontroller to receive key presses from the subject and to generate the appropriate stimulus for each trial. A randomly generated set of tactor locations was stored in the microcontroller prior to the experiment. Upon a "start" key input from the user, the microcontroller sent the random list of tactor locations one by one to the driver circuitry until a "pause" key press was received. The user responded to the tactor location by pressing one of the predefined keys on the keypad. The responses were stored in an internal EEPROM for later retrieval. The responses were chosen to be stored in the EEPROM so that all data would not be lost should a power failure occur in the middle of the experiment.

The tactor driver circuit was the same as that used in our previous experiments [9]. It consisted mainly of a power supply, a $280-\mathrm{Hz}$ oscillator, and a $16-$ Watt bridge amplifier. The main functions of the circuitry were to take a stimulus from the microcontroller, turn on the corresponding tactor for a predefined duration of $250 \mathrm{~ms}$, amplify the sinusoidal pulse to a predefined intensity of 25-30 dB sensation level (i.e., $\mathrm{dB}$ above the human detection threshold at $280 \mathrm{~Hz}$ ), and send the signal to drive the tactor. Note that the exact perceived intensities of the tactors likely varied with body locations, but all tactors were clearly perceivable.

The tactile display consists of a collection of ten tactors evenly distributed over the torso portion of a vest. Two tactors are located on each side. The distance between the upper tactor and lower tactor varied from 4-7 in. (due to height difference between subjects) for both the left and right side. The front and back both contain a tactor on each shoulder and on the lower stomach or back respectively (Fig. 2a). The distance between the right and left tactors on the front/back shoulders was $5.5 \mathrm{in}$. The distance between the upper tactors on the front/back to the lower tactor on the front/back was 9.5 in. The vest was made of a wetsuit jacket that snug-fit the wearer's body. This ensured that the tactors were always in contact with the skin. To facilitate stimulus-response compatibility, the locations of keys for the ten tactors were arranged such that they were consistent with the location of the tactors on the vest (Fig. 2b). The tactors were resonant-type vibrators made by Audiological Engineering Corp. (Somerville, MA; model VBW32; 1 in. long, 0.73 in. wide and 0.42 in. thick [11]). The sensation delivered by the tactor was similar to the vibrations felt from a commercially available massage chair.
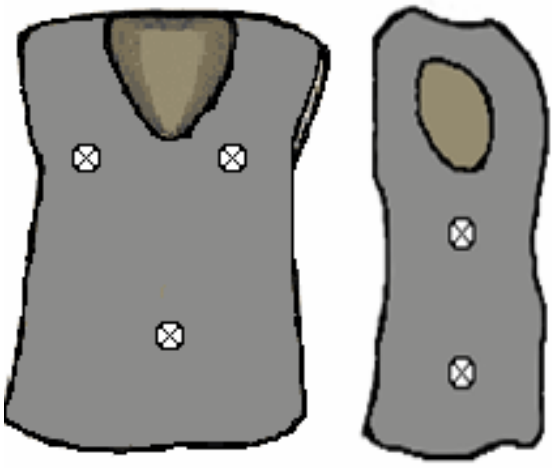

Figure 2a. Tactile Display. Shown are the front (left panel) and side (right panel) views.

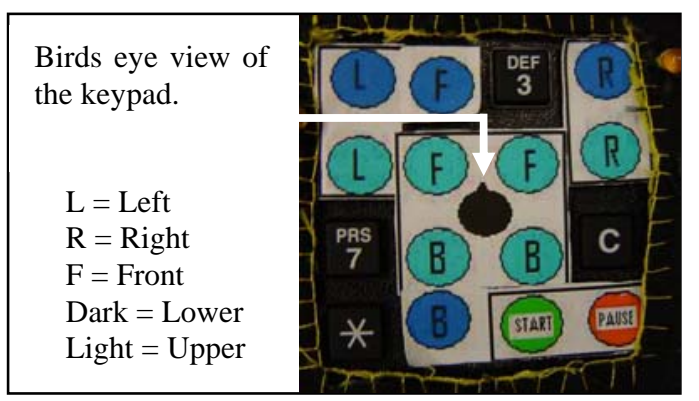

Figure 2b. Keypad as viewed by the subject.

In addition to the aforementioned hardware setup for psychophysical experiments, the ambient vibration in the KC-135A aircraft during reduced-gravity flights was also measured. The aircraft vibration data were 
acquired by attaching an accelerometer (ACH-01-03, Measurement Specialties Inc., Fairfield, NJ) to the floor of the KC-135A. The measured data was collected and stored via LabView into a laptop computer. It has been suggested that the ambient vibration aboard the KC-135A might have masked the tactors - an alternative hypothesis that could have explained why subjects performed poorly in the first flight. The acceleration data was collected during the third flight in order to test the masking hypothesis.

\subsection{Subjects}

The subjects were the four Purdue University undergraduate students who participated in the NASA Reduced-Gravity Student Flight Opportunities Program. They were between the ages of 21-23 years old. Two subjects (S1 and S2) flew on July 27, 2004, and the other two (S3 and S4) on July 28, 2004. Among the four subjects, S2 was the only one who had flown previously on the KC-135 in March 2003. All subjects gave their written consent to the experimental protocol that was approved by the IRBs at both NASA and Purdue University.

\subsection{Procedures}

For pre-flight experimentation, the subject put on the wetsuit jacket consisting of the tactile display over a thin shirt, fastened the fanny pack containing the electronics control box and batteries around the waist, and plugged the cords of the tactors into the control box. The power plugs were then attached together and the keypad was fastened to the subject's wrist. The four subjects formed two groups because NASA allowed only two students per reduced-gravity flight. In our pre-flight tests, the two subjects in the same group were tested together. At the beginning of the test, one subject laid down while the other subject remained standing. The subjects were prompted to begin and end an experimental session for twenty-five seconds (roughly the duration of zero-g during a parabola flight). To begin the session, the subject pressed the "Start" button on the keypad and a tactor was activated. The subject entered the perceived location of the activated tactor by pressing the corresponding key on the keypad. As soon as the response was registered, a new trial began. At the end of the 25-sec session, the subject pressed the "Pause" button and then entered the response for the location of the last tactor that was activated. At the end of ten simulated parabolas, the subjects switched positions and began testing again for another ten 25-second sessions. At the end of the twenty 25-sec sessions, testing ended and the subjects saved their data onto a laptop computer. This procedure was repeated twice on ground for each subject. The data obtained during the pre-flight experiments served to provide a baseline measure under the one-g gravity condition as well as to familiarize the subjects with the experimental procedures.

During the flight, the same procedure was repeated during the zero-g portion of each parabola. During the first 3 parabolas, one of the subjects (A) got acclimated with the microgravity conditions while the other subject (B) was strapped into position on the floor of the KC-135A. For parabolas \#4-13, subject B was strapped down to the floor while subject A was freefloating for experimentation. During parabola \#15, the subjects switched positions so that subject A was now strapped down to the plane floor while subject B was free-floating. Experimentation resumed for parabolas $\# 16-25$. After all the parabolas were completed, the data were saved from the control box onto the laptop computer.

While the psychophysical experiments were under way, an accelerometer recorded measurements of acceleration on the aircraft floor. When the subjects were getting ready for the psychophysical experiment, they pressed a button on the laptop computer to begin recording data from the accelerometer. At the end of the experimentation portion of the flight, a button was pressed again on the laptop to end data collection.

It should be noted that all subjects took the prescription drug SCOP-DEX in order to reduce sickness in microgravity. In order to assess the possible effect of the drug on vibrotactile perception, post-flight data was gathered on subjects S3 and S4 right after their flight. The procedure was the same as that used in the pre-flight testing.

\subsection{Data Analysis}

The responses from each subject were downloaded directly from the microcontroller into a text file. The percent-correct score was calculated for both the low and high cognitive-load conditions for each subject. Average percent-correct scores were then computed separately for the strapped down and free-floating conditions, respectively. A two sampled proportion test was then performed on the percent-correct scores of the two conditions for the group. Since enough trials were obtained, the sampling distribution of the data obtained follows a normal, also known as a Gaussian, distribution [12]. 


\section{Results}

The preflight results are shown in Table 1 . As expected, the percent-correct scores were quite high when the subjects were tested in a laboratory setting. The scores from the "lying down" and the "standing up" conditions were essentially the same $(88.5 \%$ and $90 \%$, respectively), since there was no difference in the cognitive load associated with the two body postures in one-g. To investigate why the preflight accuracy were not nearly perfect, we looked at the stimulus-response confusion patterns. For both the lying down and standing up conditions, most errors occurred when the subject misjudged a lower tactor on either side of the torso to be in the higher position (ref. Fig. 2a, right panel). Curiously, the higher tactors were rarely judged to be in the lower positions.

Table 1. Preflight data

\begin{tabular}{|c|c|c|c|c|}
\hline & \multicolumn{2}{|c|}{ Lying Down } & \multicolumn{2}{c|}{ Standing Up } \\
\hline Subject & $\begin{array}{c}\text { No. of } \\
\text { Trials }\end{array}$ & $\begin{array}{c}\% \\
\text { Correct }\end{array}$ & $\begin{array}{c}\text { No. of } \\
\text { Trials }\end{array}$ & $\begin{array}{c}\% \\
\text { Correct }\end{array}$ \\
\hline S1 & 418 & 79.7 & 400 & 75.3 \\
\hline S2 & 400 & 92.5 & 409 & 95.1 \\
\hline S3 & 383 & 97.4 & 392 & 96.7 \\
\hline S4 & 158 & 93.0 & 183 & 85.2 \\
\hline Average & & $\mathbf{9 0}$ & & $\mathbf{8 8 . 5}$ \\
\hline
\end{tabular}

* Note that some of S4's data were corrupted; therefore the number of trials for this subject was relatively small.

Experimental data obtained from the actual flight, where subjects experienced microgravity conditions, is shown in Table 2. The average in-flight percentcorrect score (82.9\%) was only slightly lower than the preflight data (88.5-90\%). There was a significant difference between the data obtained in the low and high cognitive-load conditions (82.9\% and $47.4 \%$, respectively). In order to determine the statistical relevancy of these values, the statistical analysis method known as the two proportions test was applied to the percent-correct scores of the low and high cognitive-load conditions. This analysis yielded a pvalue of zero, which means that the null hypothesis that the percent-correct scores for the two conditions were the same should be rejected [12]. The results therefore strongly supported the hypothesis that an increase in cognitive load adversely affected the subjects' ability to locate vibrotactile stimulation on the body surface. Note that S2's scores in the freefloating condition (63.0\%) were much higher than those from the other three subjects. Recall that S2 had flown aboard a KC-135A prior to this experiment. It is quite possible that as one becomes accustomed to the microgravity environment, the cognitive load associated with flying in zero-g decreases. Therefore, S2 was able to devote more attention to the tactors and performed better than the other subjects.

Table 2. In-flight data

\begin{tabular}{|c|c|c|c|c|}
\hline & \multicolumn{2}{|c|}{ Strapped Down } & \multicolumn{2}{c|}{ Free-floating } \\
\hline Subject & $\begin{array}{c}\text { No. of } \\
\text { Trials }\end{array}$ & $\begin{array}{c}\% \\
\text { Correct }\end{array}$ & $\begin{array}{c}\text { No. of } \\
\text { Trials }\end{array}$ & $\begin{array}{c}\% \\
\text { Correct }\end{array}$ \\
\hline S1 & 196 & 74.5 & 160 & 40.6 \\
\hline S2 & 172 & 91.3 & 92 & 63.0 \\
\hline S3 & 151 & 90.1 & 78 & 47.4 \\
\hline S4 & 90 & 73.3 & 29 & 34.5 \\
\hline Average & & $\mathbf{8 2 . 9}$ & & $\mathbf{4 7 . 4}$ \\
\hline
\end{tabular}

Results obtained form the post-flight tests were similar to those obtained in the preflight testing. The average percent-correct scores for the "lying down" and "standing up" conditions were nearly identical (90.7\% and 93.4\%, respectively). The results indicated that the prescription drug SCOP-DEX did not affect subjects' vibrotactile perception.

Table 3. Post flight data for S3 and S4 while still under effects of medication

\begin{tabular}{|c|c|c|c|c|}
\hline & \multicolumn{2}{|c|}{ Lying Down } & \multicolumn{2}{c|}{ Standing Up } \\
\hline Subject & $\begin{array}{c}\text { No. of } \\
\text { Trials }\end{array}$ & $\begin{array}{c}\% \\
\text { Correct }\end{array}$ & $\begin{array}{c}\text { No. of } \\
\text { Trials }\end{array}$ & $\begin{array}{c}\% \\
\text { Correct }\end{array}$ \\
\hline S3 & 214 & 96.3 & 211 & 98.1 \\
\hline S4 & 182 & 84.1 & 211 & 88.6 \\
\hline Average & & $\mathbf{9 0 . 7}$ & & $\mathbf{9 3 . 4}$ \\
\hline
\end{tabular}

Finally, the results of acceleration recordings on the floor of KC-135A indicated that there was little vibration inside the aircraft presumably due to the one inch thick foam rubber padding on all surfaces. The vibration recordings resulted in white noise comprised of a broad range of frequencies barely detectable by the accelerometer.

\section{Discussion}

In this study, which was the third in a series of experiments on vibrotactile perception in alteredgravity environment, we investigated the effect of cognitive load on subjects' ability to locate vibrotactile stimulation on the body surface. The preflight results showed that lying down or standing up produced similar results on tactor location identification. This was to be expected since maintaining either position in a laboratory setting (one-g) required little effort on the subjects' part, and therefore demanded similar and minimal amounts of cognitive load. The in-flight 
results showed that subjects performed significantly better while being strapped to the floor of the aircraft than while flying freely in microgravity. Since subjects had to devote significant amount of attention to body orientation and movement while free-flying in microgravity, the results were interpreted as indicating that an increase in cognitive load was the main cause of the decrease in tactor-location identification performance in microgravity. An alternative interpretation could have been that the subjects performed better while being strapped down because they had access to a fixed visual reference frame. However, this was unlikely the cause of the observed performance difference between the strapped-down and free-flying conditions because subjects had to look at the keypad at all times in order to push the appropriate buttons for responses. Therefore, our results offer an explanation in terms of cognitive load for why the subjects in the first flight performed significantly worse in zero-g than in one-g.

Data was also taken from subjects while they were still under the influence of an anti-nausea drug. It was concluded that the use of the drug had little effect on vibrotactile perception. Taken together, of the six average percent-correct scores obtained during the current study (pre-flight, in-flight, post-flight, each with two conditions), only the score measured during zero-g flight under the free-floating condition (47.4\%) was significantly lower than the rest of the scores. Since this was the only truly high cognitive-load condition tested, we conclude that cognitive-load indeed affected the ability to locate vibrotactile stimulations accurately.

However, our results should not be taken as an indication that haptic displays are not suited for microgravity environments. We used a microgravity environment to induce a high cognitive load condition for subjects who were not experienced with the zero-g environment. As a user becomes acquainted with moving in zero-g, the cognitive load associated with free-floating will decrease over time. Therefore we expect users more experienced with the zero-g environment to perform better than our subjects did. Indeed, S2's in-flight data pointed in that direction.

Our results provide strong evidence that cognitive load was the main cause for the relatively poor performance during the first flight. In addition, we have learned that, like other sensory modalities, the somatosensory system also suffers from the effects of attention and cognitive load. The results from all three flights in this series of investigation have implications for the design of haptic interfaces in general. As a user engages in multitasking, there is a cost associated with the user's performance with a multimodal interfaces.
The challenge for the interface designer is to make an interface as intuitive as possible so as to minimize the cognitive load associated with the use of a multimodal interface.

\section{Acknowledgment}

Our participation in the NASA Reduced Gravity Student Flight Opportunities Program would not have been possible without the generous support of the Schools of Electrical and Computer Engineering, Industrial Engineering, and Aeronautical Engineering, and the Dean's Office of the College of Engineering at Purdue University. We are also grateful to our corporate sponsors BAE Systems and Lockheed Martin. In addition, this work was partially funded by a National Science Foundation Faculty Early Career Development (CAREER) Award under Grant No. 9984991-IIS. The authors wish to thank NASA for this wonderful experience.

\section{References}

[1] Air Force Research Lab Site Spatial Disorientation Countermeasures. Available: $<$ http://www.spatiald.wpafb.af.mil/index.asp>

[2] Naval Aerospace Medical Research Laboratory TSAS: Accurate Orientation Information through a Tactile Sensory Pathway in Aerospace, Land and Sea Environments. $<$ http://www.namrl.navy.mil/accel/tsas/index.htm>

[3] MIT Man Vehicle Laboratory. Available: $<$ http://mvl.mit.edu/MVL-research.htm>

[4] A. H. Rupert, "An instrument solution for reducing spatial disorientation mishaps - A more "natural" approach to maintaining spatial orientation," IEEE Engineering in Medicine and Biology Magazine, vol. 19, 2000, pp. 71-80.

[5] R. W. Cholewiak and A. A. Collins, "The generation of vibrotactile patterns on a linear array: Influences of body site, time, and presentation mode," Perception \& Psychophysics, vol. 62, pp. 1220-1235, 2000.

[6] H-J. van Veen, M. Spape, J. van Erp, "Waypoint navigation on land: different ways of coding distance to the next waypoint," Proceedings of EuroHaptics 2004, pp. 160-5, 2004.

[7] H. Z. Tan, R. Gray, J. J. Young, and R. Traylor, "A haptic back display for attentional and directional cueing," Haptics-e: The Electronic Journal of Haptics Research, vol. 3, pp. 20 pp, 2003.

[8] F. A. Geldard, Sensory Saltation: Metastability in the Perceptual World. Hillsdale, New Jersey: Lawrence Erlbaum Associates, 1975.

[9] R. Traylor and H. Z. Tan, "Development of a wearable haptic display for situation awareness in altered-gravity environment: Some initial findings," Proceedings of the International Symposium on Haptic Interfaces for Virtual Environment and Teleoperator Systems, pp. 259-164, 2002. 
[10] Adcock, Amy B. Effects of Cognitive Load on Processing and Performance. Available: http://aimlab.memphis.edu/amyscogpaper.pdf

[11] Audiological Engineering Corps. Site of Tactaid and Tactilator. Available http://store.yahoo.com/audiologicalengineering/

[12] D. R. Anderson, D. J. Sweeney, and T. A. Williams. Statistics for Business and Economics, $8^{\text {th }}$ Edition. Cincinnati, Ohio. South-Western, 2002. 